PROC. OF JSCE,

No. 272, APR. 1978

\title{
CONSTITUTIVE THEORY FOR SOLID-FLUID MIXTURE AND ITS APPLICATION TO STRESS WAVE PROPAGATION THROUGH COHESIVE SOIL
}

\author{
By Fusao OKA*
}

\section{INTRODUCTION}

In order to clarify the ground motion under dynamic loading such as earthquakes, the realistic constitutive relation of soil should be obtained. The essential nature of soil is that soil is considered as the multi-phase mixture and has a non-linear property. One of the purpose of this paper is to construct the solid-fluid mixture theory for saturated cohesive soil.

Many authors treated the saturated soil as the solid-fluid mixture. Biot ${ }^{1)}$ developed the threedimensional theory of saturated porous elastic material and applied it to the stress wave propagation problem. Ishihara ${ }^{2)}$ proposed the theory of porous material having the heat effect which is considered as the generalization of Biot's theory. Ishihara introduced the saturated porous material with the heat effect from the linear irreversible thermodynamics on the basis of Onsager's reciprocal theorem. Using the Onsager's relation, he formulated the flow through the porous solid and the heat diffusion. The theory obtained as the results includes the poroelasticity and the thermoelasticity proposed by Biot.

As Truesdell ${ }^{3)}$ pointed, Onsager's relation has the following defects.

1. Generally the resolution of entropy production rate to force and flux is not uniquely determined.

2. Onsager's relation is strictly limited to linear process.

From the above discussion, the theory based on the Onsager's relation is too restrictive for

* M.S.C.E., Research Associate of Transportation Engng., Kyoto University. continuum mechanics. The non-equilibrium thermodynamic theory proposed by Coleman $\& \mathrm{Noll}^{4)}$ is not restricted to the state near the equilibrium state and the linear process. Using this theory, Green \& Naghdi, ${ }^{5)}$ Ingram \& Eringen ${ }^{6)}$ and Bowen $^{7}$ et al. investigated the mixture. Müller ${ }^{8)}$ proposed the more rational approach to a mixture by his original thermodynamics based on the modern continuum mechanics developed by Coleman \& Noll. ${ }^{4)}$

Ishihara ${ }^{9)}$ clarified the physical meaning of coefficients in the Biot's equation and showed the similarity of Biot's theory to the linear viscoelasticity. So, Akai \& Hori $^{10)}$ considered that the visco-elastic nature of soil is due to the solid-fluid interaction. But Ishihara concluded that in the actual situation such as earthquakes, the attenuation of compressional wave due to the friction between the solid and fluid is close to zero. After all, the inelasticity existing inherently in the solid phase is more important for energy absorption than the friction due to the interaction between the solid and fluid during the earthquakes. Adachi $^{11}$ induced the theories of mixture constituting of elastic solid and two or more fluids and elastic-plastic solid and viscous fluid. But, he did not apply them to the practical problem in soil mechanics.

In this paper, the saturated cohesive soil is formulated as the mixture of an elastic fluid and the viscoelastic-viscoplastic solid. The author previously proposed the constitutive theory ${ }^{12}$ ) that can explain the behavior of a normally consolidated clay, but there are still left difficulties that the theory is not sufficient to explain the behavior during the unloading. By the Fourier transformation of the wave form which is obtained by stress wave propagation test at low stress level, a viscoelasticity seems to be also the 
properties of cohesive soil. So, the constitutive theory for cohesive soil must be able to explain the visco-elastic behavior. From the above discussion, the inelasticity in the solid phase is assumed to be viscoelastic-viscoplastic.

The concrete constitutive theory is induced by introducing internal state variables.

The mechanical nature of principle of effective stress has not been discussed sufficiently. In the present stage, Terzaghi's effective stress is mainly dependent of the experimental results rather than rational interpretations. Following the mixture theory, the author can explain the meaning of effective stress concept clearly. It can be seen that effective stress concept is usefull during the dynamic loading.

In section 2, experimental results that are obtained by the Fourier transform of experimental test data are shown. In section 3 , the mixture theory of solid and fluid is presented. In section 4 , the constitutive theory for a normally consolidated clay is proposed including the viscoelastic and viscoplastic property. In section 5, one-dimensional stress wave propagation through cohesive soil is discussed. Numerical results are given by integrating the differential relation along the characteristics numerically.

\section{EXPERIMENTAL STUDY}

\subsection{Introduction}

The author has carried on the stress wave propagation test in order to investigate the dynamic characteristics of soil, using the special triaxial cell connected to the shock tube. The test procedure has been presented in the previous papers. ${ }^{10), 12}$ The pressure form given by experiment is pulsative and contained the wide range frequency components. In this paper, a pulsative wave form is replaced by the combination of harmonic wave and the character of dispersion is clarified by discussing the phase velocity and attenuation coefficient. The soil sample is a Fukakusa silty clay consolidated under the pressure of $2.0 \mathrm{~kg} / \mathrm{cm}^{2}$ for two months. The liquid limite is $57.5-60.5 \%$ and the plastic limite is 28.1 and pre-consolidated pressure is $0.65-0.85 \mathrm{~kg} / \mathrm{cm}^{2}$. Peak stress wave is $0.1-1.0 \mathrm{~kg} / \mathrm{cm}^{2}$. It is assumed that stress wave is smooth and can be replaced by the combination of harmonic wave.

$$
\Sigma=A \exp \{i[\omega t+(k+i \alpha) x]\}
$$

where, $\Sigma$ is stress, $A$ is its amplitude, $\omega$ is the angular frequency, $k$ is the wave number, $\alpha$ is the attenuation coefficient, $t$ is the time, $i$ is a imaginary unit and $x$ is the coordinate of position. $\Sigma$ is transformed by Fourier transformation to $\Sigma^{\prime}$.

$$
\begin{aligned}
\Sigma^{\prime} & =\frac{1}{T} \int_{-T / 2}^{T / 2} \sum \exp (i \omega t) d t \\
& =A \exp \left(-\alpha x+i \frac{\omega x}{C_{p}}\right) \quad \cdots \cdots \cdots \cdots \cdots \cdots \cdots \cdots(1) \\
\alpha= & -\frac{1}{R} \ln \left[\Sigma_{2}{ }^{\prime}(i \omega) / \Sigma_{1}{ }^{\prime}(i \omega)\right], \quad C_{p}=\omega \frac{R_{2}-R_{1}}{\theta_{2}-\theta_{1}}
\end{aligned}
$$

where $C_{p}$ is phase velocity, $R_{2}-R_{1}$ is the distance between two soil stress gages, $\theta_{2}-\theta_{1}$ the difference of phase angle between two observation points, $T$ is a time interval, $R_{i}$ shows the position of the stress gauge $(i=1$ or 2$)$ and Number 1 and 2 show the two different positions.

\subsection{Phase velocity}

Fig. 1 shows the spectrum of stress wave. From this figure, the predominant frequency of the stress wave observed in stress wave propagation test is $0-200 \mathrm{cps}$. Fig. 2 shows the relationships between phase velocity and frequency. In this figure, $C_{0}$ is a speed of wave front. From this figure, in the higher frequency range than $300-350 \mathrm{cps}$, phase velocity exceeds the speed of the wave front and increases. Generally, in the linear viscoelastic body which has an instantaneous elasticity, phase velocity asymptotically approaches elastic wave velocity as the frequency becomes large. But, in the linear Voigt type viscoelastic body, phase velocity exceeds the wave velocity $C_{0}$ and increases as frequency becomes large. From the above consideration and Fig. 2, it seems to be that the elastic property

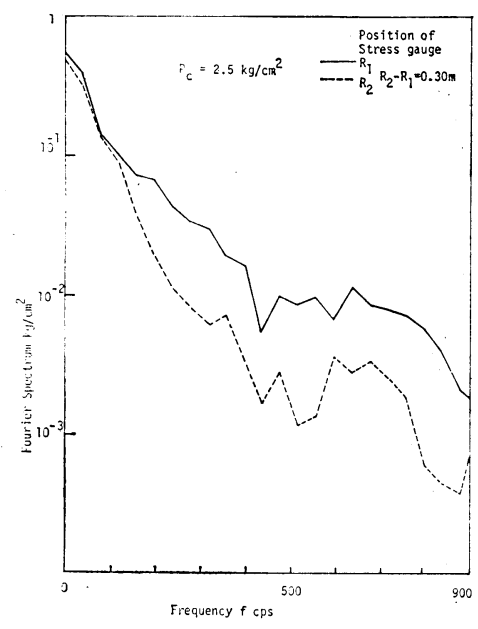

Fig. 1 Fourier Spectrum. 


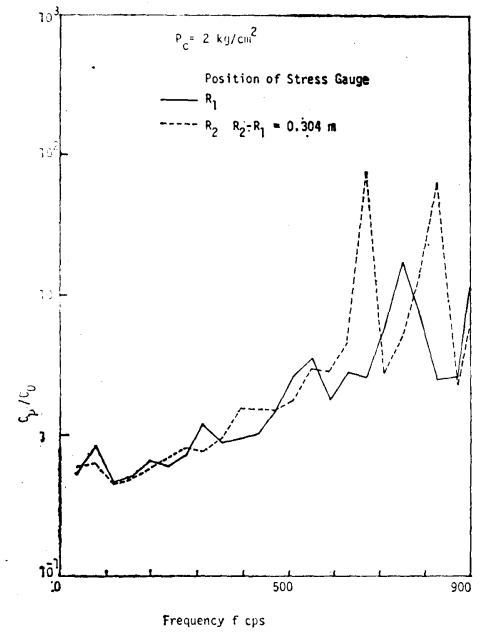

Fig. 2 Relationship between Phase velocity and Frequency.

of cohesive soil is described by a linear Voigt model.

\subsection{Attenuation coefficient}

The attenuation of stress during wave propagation is estimated by attenuation coefficient $\alpha$. The two waves as samples are equal in peak stress. Figs. 3 and 4 show the relation between attenuation coefficient $\alpha$ and frequency. From

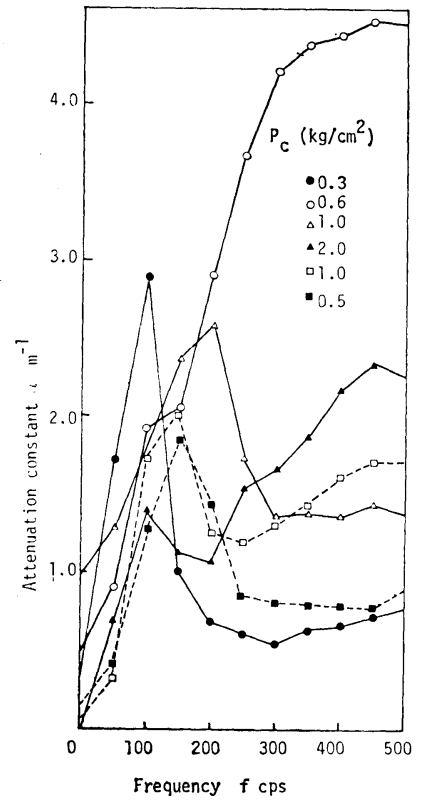

Fig. 3 Relationship between Attenuation constant $\alpha$ and Frequency.

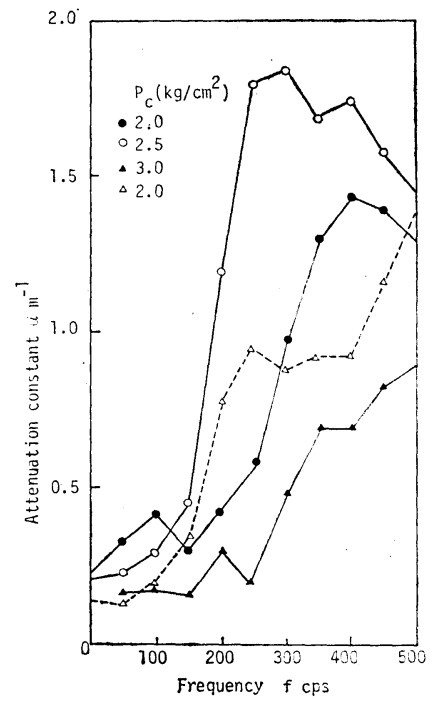

Fig. 4 Relationship between Attenuation constant $\alpha$ and Frequency.

these figures, it can be seen that attenuation coefficient becomes large as the frequency increases. $\alpha$ begins to increase at almost $0 \mathrm{cps}$ in Fig. 3, but in Fig. 4 at almost $100 \mathrm{cps}$.

Fig. 4 is the case of high confining pressure $P_{c}$. The tendency of increase of $\alpha$ is not monotonous, but has extreme value between $150 \mathrm{cps}$ and 400 cps. As a confining pressure becomes large, the frequency at which $\alpha$ is extremum increases. Furthermore, $\alpha$ decreases as the confining pressure becomes large.

It can be concluded that the viscoelastic property of cohesive soil is approximately described by a linear spring-Voigt model from above discussion between $0 \mathrm{cps}$ and $100 \mathrm{cps}$.

From the dynamical test results for the cohesive soil, Kondner \& Ho, ${ }^{13}$ Nishigaki \& Hirobe $^{14)}$ and Hatano \& Watanabe ${ }^{15)}$ reported that the elastic shear modulus becomes large with the increase of input frequency. Furthermore, they concluded that the dynamical behavior of cohesive soil can be approximately described by a linear spring-Voigt model. In contrast to these researchers, Krizek \& Franklin ${ }^{16)}$, Hara ${ }^{17)}$ and Parmelee et al. ${ }^{18)}$ showed that the dynamical behavior of some soft clay does not depend on the frequency. After all, viscoelastic property of cohesive soil depends on the material property and the level of strain. So, in some soils, viscoelastic property is predominant and in the other viscoelastic behavior can be neglected. In order to reduce a general constitutive equation of 
cohesive soil, the viscoelastic property must be considered.

\section{THEORY OF SOLID-FLUID MIXTURE}

\subsection{Introduction}

The review of the theory of mixture is reported by Truesdell ${ }^{3)}$ and Atkins \& Craine. ${ }^{19)}$ Here, the following two important points of dispute in the theory of mixture is discussed.

1) The definition of total stress

2) Entropy production inequality

Modern treatment of mixture was firstly tried by Truesdell. $^{20)} \mathrm{He}$ introduced three metaphysical principles.

1) All properties of the mixture must be mathematical consequences of properties of the constituents.

2) So as to describe the motion of a constituent, we may in imagination isolate it from the rest of the mixture, provided we allow properly for the actions of the other constituents upon it.

3) The motion of the mixture is governed by the same equations as is a single body.

From the third principle, total stress, total energy and total body force etc. are defined. He defined the total stress tensor as follows.

$$
\begin{aligned}
t_{i j}= & \sum_{a} t_{i j}-\sum_{a} \rho_{a} u_{i} u_{j} \\
t_{i j}: & \text { total stress tensor acting on the surface } \\
& \text { of mixture } \\
u_{i}: & \text { diffusion velocity vector } \\
t_{i j}: & \text { partial stress tensor }
\end{aligned}
$$

From Truesdell to Müller or Green \& Naghdi, ${ }^{21)}$ the theory of mixture has been confirmed by applying the theory to the results in classical thermo-chemistry, for example, Dalton's Law. Against the Truesdell's definition, Green and Naghdi defined the total stress by Eq. (3).

$$
t_{i j}=\sum_{a} t_{i j}
$$

Green \& Naghdi construct the theory of mixture based on the different primitive concept. They took as the fundamental postulate the balance equation not for the several constituents, but for the mixture as a whole. When we face to the boundary value problem including the total stress as the boundary condition, it is important which definition is used. As Atkins \& Craine pointed out, for the general mixture rather than ideal gas need not be described by Eqs. (2) or (3). Müller and Bowen ${ }^{7)}$ criticized that the Green \& Naghdi's theory ${ }^{5)}$, because their theory was not consistent with the classical thermo-chemical results when it was applied to the ideal gas mixture in equilibrium state. In order to solve the dilemma, Green \& Naghdi ${ }^{21)}$ asserted that the partial stress in the classical theory is different from that in their theory. After that time, they recognized the error and introduced the arbitrary function which did not violate the balance law as a whole in order to consist with the classical results. Furthermore, they took the energy equation for several constituents to introduce the arbitrary function ${ }^{22)}$. The usefulness of the method in which the arbitrary function is introduced is based on the thermodynamics by them. ${ }^{23)}$ Müller ${ }^{8)}$ proposed the theory of mixture that consisted with the result of classical gas mixture and was well motivated physically. His assertion is based on the two important postulates. One of them is for the independent variable in the constitutive assumption and the other is for the entropy production inequality. Introducing the density gradient as the independent variable, Müller prevents the theory from oversimplifying. This method solved the dilemma into which Bowen fell. ${ }^{7}$ He took the entropy flux as the constitutive quantity which was specified by a constitutive equation, not by the Eq. (4).

$$
\text { entropy flux }=(\text { heat flux }) /(\text { temperature })
$$

On the basis of the several principle (equipresence, objectivity, entropy inequality, postulate for entropy supply and constitutive equation for entropy flux), Eq. (4) is derived for a broad class of single material by Müller. Truesdell ${ }^{3)}$ concluded that the only Müller's theory seemed to satisfy the general principle of modern continuum mechanics. Comparing with the Müller's theory, Green \& Naghdi's one is less sensitive and has the advantage that total stress can take the form that is applicable to the practical problem. Fundamentally, there is no difference between the basic equations in the Müller's theory and Green \& Naghdi's one. In the case of treating the motion of saturated soil, the motion of soil skeleton is more important for engineering problem than that as a single material. So, we need not describe the mean motion. The author induces the theory of mixture from the point of view of Green \& Naghdi basically.

\subsection{Balance law for two phase mixture}

Here, soil is considered as a mixture of two interacting constituents each of which is regarded as a continuum. It is assumed that each point is occupied simultaneously by all constituents. 
The position at the peculiar particle of constituents at time $\tau$ is denoted by $x_{i}^{(\alpha)}(\tau)$.

$$
\begin{aligned}
& x_{i}^{(\alpha)}=\hat{x}_{i}^{(\alpha)}\left(X_{1}^{(\alpha)}, X_{2}^{(\alpha)}, X_{3}^{(\alpha)}, \tau\right) \quad(-\infty<\tau \leq t) \\
& \alpha=f \text { or } s
\end{aligned}
$$

The indices $(f)$ and $(s)$ denote the fluid phase and solid phase respectively. $x_{i}{ }^{(\alpha)}$ is a reference position of each particle. At time $t$, any position is occupied by a particle of each constituent.

$$
x_{i}^{(s)}=x_{i}{ }^{(f)}=x_{i}
$$

\section{Balance of mass}

It is assumed that a mass of an individual constituent is conserved. So, we postulate following balance equation for each constituent.

$$
\begin{aligned}
& \int_{s} \bar{\rho}^{s} v_{i} n_{i} d s+\int_{v} \frac{\partial \bar{\rho}^{s}}{\partial t} d v=0 \\
& \int_{s} \bar{\rho}^{f} v_{i} n_{i} d s+\int_{v} \frac{\partial \bar{\rho}^{f}}{\partial t} d v=0 \\
& \bar{\rho}^{s}=(1-n) \rho^{s} \ldots \ldots \ldots \ldots \ldots \ldots \ldots \ldots \ldots \ldots \ldots \ldots \ldots \ldots \ldots \ldots \ldots \ldots \ldots \ldots
\end{aligned}
$$

$\rho^{s}$ and $\rho^{f}$ are the specific mass densities of soil particle and water respectively. $\bar{\rho}^{s}$ and $\bar{\rho}^{f}$ are the mass densities of each continuum constituent (solid and fluid) constituting of the mixture. $n$ is the porosity of the mixture. $v_{i}$ is the component of velocity vector. $v$ is the arbitrary volume and $s$ is its surface.

Balance of linear momentum

The balance equations for linear momentum for each constituent are taken as follows, For solid phase,

$$
\begin{gathered}
\int_{s} t_{i j}^{s} n_{j} d s-\int_{v}\left(\pi_{i}-b_{i}{ }^{s}\right) d v=\int_{s} \bar{\rho}^{s} v_{i}{ }^{s} v_{j}{ }^{s} n_{j} d s \\
\quad+\int_{v} \frac{\partial}{\partial t}\left(\bar{\rho}^{s} v_{i}{ }^{s}\right) d v \quad \ldots \ldots \ldots \ldots \ldots \ldots \ldots \ldots \ldots \ldots \ldots \ldots \ldots \ldots \ldots \ldots \ldots \ldots \ldots \ldots \ldots
\end{gathered}
$$

For fluid phase,

$$
\begin{aligned}
& \int_{s} t_{i j}^{f} n_{j} d s+\int_{v}\left(\pi_{i}+b_{i}{ }^{f}\right) d v=\int_{s} \bar{\rho}^{f} v_{i}{ }^{f} v_{j}{ }^{f} n_{j} d s \\
& \quad+\int_{v} \frac{\partial}{\partial t}\left(\bar{\rho}^{f} v_{i}{ }^{f}\right) d v \ldots \ldots \ldots \ldots \ldots \ldots \ldots \ldots \ldots \ldots \ldots \ldots \ldots \ldots \ldots \ldots \ldots \ldots \ldots \ldots
\end{aligned}
$$

where $t_{i j}^{(\alpha)}$ is a partial stress tensor or bulk area averaged stress tensor. Total stress is defined by Eq. (11).

$$
t_{i j}=t_{i j}^{s}+t_{i j}^{f}
$$

If $u$ is a water pressure in the void, Terzaghi's effective stress tensor is denoted by $t_{i j}^{e}$.

$$
\begin{aligned}
t_{i j}^{e} & =t_{i j}-u \delta_{i j}=t_{i j}^{s}-(1-n) u \delta_{i j} \\
t_{i j}^{f} & =n u \delta_{i j}
\end{aligned}
$$

The definition of Terzaghi's effective stress tensor is discussed further later. $\pi_{i}$ is the component of interaction force vector arising from the transfer of momentum between constituents. $b_{i}(\alpha)$ is the component of external body force vector. Total body force $b_{i}$ is the sum of $b_{i}{ }^{s}$ and $b_{i}{ }^{f}$.

From Eqs. (9) and (10), linear momentum is also balanced for mixture as a whole. Using the Eqs. (5) and (12), the local forms of Eqs. (9) and (10) are denoted as follows if functions in the equations are continuous.

$$
\begin{aligned}
\frac{\partial t_{i j}^{e}}{\partial x_{j}} & =\bar{\rho}^{s} \frac{d v_{i} s}{d t}-\frac{\partial(1-n) u \delta i j}{\partial x_{j}}+\pi_{i}-\bar{\rho}^{s} b_{i} s \\
\frac{\partial t_{i j}^{f}}{\partial x_{j}} & =\bar{\rho}^{f} \frac{d v_{i}{ }^{f}}{d t}-\pi_{i}-\bar{\rho}^{f} b_{i} f \ldots \ldots \ldots \ldots \ldots \ldots \ldots \ldots \ldots \ldots
\end{aligned}
$$

where

$$
\frac{d v_{i}^{\alpha}}{d t}=\frac{\partial v_{i}^{\alpha}}{\partial t}+v_{i}{ }^{\alpha} \frac{\partial v_{i}^{\alpha}}{\partial x_{j}} \quad(\alpha=s \text { or } f)
$$

\section{Balance of angular momentum}

Balance equation of angular momentum reffered to a fixed place for individual constituent is assumed in the form.

$$
\begin{aligned}
& \frac{\partial}{\partial t} \int_{v} e_{i j k} \bar{\rho}^{s} v_{i}{ }^{s} x_{j} d v+\int_{s} e_{i j k} \bar{\rho}^{s} v_{i} v_{m_{m}}{ }^{s} x_{j} n_{m} d s \\
& \quad+\int_{v} e_{i j k}\left(\pi_{i}-\bar{\rho}^{s} b_{i}{ }^{s}\right) x_{j} d v-\int_{s} e_{i j k} t_{m i}^{s} n_{m} x_{j} d s=0
\end{aligned}
$$

where, $e_{i j k}$ is a permutation symbol.

From Eqs. (14) and (16), we get following equation for solid phase.

$$
e_{i j k} t_{j i}^{s}=0 \quad t_{i j}^{s}=t_{j i}^{s}
$$

Similarly, for fluid phase, Eq. (18) is obtained.

$$
t_{i j}^{f}=t_{j i}^{f}
$$

From above consideration, partial stress for each constituent is symmetric.

\section{Balance of energy}

It is sufficient to assume that each constituent has a common temperature. So, we postulate the balance equation of energy for a mixture as a whole in the form Eq. (19).

$$
\begin{aligned}
& \frac{\partial}{\partial t} \int_{v}\left(\bar{\rho}^{s} \varepsilon^{s}+\bar{\rho}^{f} \varepsilon^{f}+\frac{1}{2} \bar{\rho}^{s} v_{i} v_{i} v^{s}+\frac{1}{2} \bar{\rho}^{f} v_{i}^{f} v_{i}^{f}\right) d v \\
& \quad+\int_{s}\left[\left(\bar{\rho}^{s} \varepsilon^{s}+\frac{1}{2} \bar{\rho}^{s} v_{i} v_{i} s\right) v_{j}^{s}\right. \\
& \left.\quad+\left(\bar{\rho}^{f} \varepsilon^{f}+\frac{1}{2} \bar{\rho}^{f} v_{i} v_{i} v^{f}\right) v_{j}^{f}\right] n_{j} d s \\
& =\int_{s}\left(t_{i j}^{s} v_{i}^{s}+t_{i j}^{f} v_{i}^{f}\right) n_{j} d s+\int_{s}\left(q_{i}^{s}+q_{i}^{f}\right) n_{i} d s \\
& \quad+\int_{v}\left(\bar{\rho}^{s} s^{s}+\bar{\rho}^{f} s^{f}\right) d v+\int_{v}\left(\bar{\rho}^{s} b_{i} v_{i} v^{s}+\bar{\rho}^{f} b_{i}{ }^{f} v_{i}^{f}\right) d v
\end{aligned}
$$

where $\varepsilon^{\alpha}$ is internal energy density, $q_{i}^{\alpha}$ is heat influx vector, $s^{\alpha}$ is heat supply. $(\alpha=s$ or $f)$ In the local form, Eq. (19) is rewritten by 


$$
\begin{gathered}
\bar{\rho}^{s} \frac{d \varepsilon^{s}}{d t}+\bar{\rho}^{f} \frac{d \varepsilon^{f}}{d t}=t_{i j}^{s} v_{i, j}^{s}+t_{i j}^{f} v_{i, j}^{f}-\bar{\rho}^{s} v_{i} s \frac{d v_{i}^{s}}{d t} \\
-\bar{\rho}^{f} v_{i} \frac{d v_{i}^{f}}{d t}+t_{i j, j}^{s} v_{i}^{s}+t_{i j, j}^{f} v_{i}^{f}+q_{i, i}^{s}+q_{i, i}^{f} \\
\quad+\bar{\rho}^{s} s^{s}+\bar{\rho}^{f} s^{f}+\bar{\rho}^{s} b_{i} v_{i_{i}}{ }^{s}+\bar{\rho}^{f} b_{i}{ }^{f} v_{i}^{f} \quad \ldots \ldots \ldots \ldots
\end{gathered}
$$

Using the Eqs. (12), (14) and (15), following Eq. (21) is given in local form.

$$
\begin{aligned}
& \bar{\rho}^{f} \frac{d \varepsilon^{f}}{d t}+\bar{\rho}^{s} \frac{d \varepsilon^{s}}{d t}=t_{i j}^{e} v_{i, j}^{s}+(1-n) u \delta_{i j} v_{i, j}^{s}+t_{i j}^{f} v_{i, j}^{f} \\
& \quad+q_{i, i}^{f}+q_{i, i}^{s}+\bar{\rho}^{f} s^{f}+\bar{\rho}^{s} s^{s}-\pi_{i}\left(v_{i} f-v_{i}^{s}\right)
\end{aligned}
$$

\section{Entropy production inequality}

We assume the following entropy production inequality for mixture as a whole, which is equivalent to one used by Truesdell.

$$
\begin{aligned}
& \int_{v} \sigma d v=\frac{\partial}{\partial t} \int_{v}\left(\bar{\rho}^{s} \eta^{s}+\bar{\rho}^{f} \eta^{f}\right) d v \\
& +\int_{s}\left(\bar{\rho}^{s} \eta^{s} v_{i}{ }^{s}+\bar{\rho}^{f} \eta^{f} v_{i}^{f}\right) n_{i} d s-\int_{s} \frac{1}{\theta}\left(q_{i}^{s}+q_{i}^{f}\right) n_{i} d s \\
& \quad-\int_{v} \frac{1}{\theta}\left(\bar{\rho}^{s} s^{s}+\bar{\rho}^{f} s^{f}\right) d v \geqq 0 \ldots \ldots \ldots \ldots \ldots \ldots \ldots(22)
\end{aligned}
$$

$\eta^{\alpha}$ is an entropy per unit mass and $\sigma$ is an entropy production density of a mixture as a whole, and $\theta$ is a absolute temperature.

From Eqs. (21) and (22), following reduced inequality in the local form is obtained.

$$
\begin{gathered}
\bar{\rho}^{s} \frac{d \eta^{s}}{d t}+\bar{\rho}^{f} \frac{d \eta^{f}}{d t}-\frac{1}{\theta}\left(\bar{\rho}^{s} \frac{d \varepsilon^{s}}{d t}+\bar{\rho}^{f} \frac{d \varepsilon^{f}}{d t}\right) \\
+\frac{1}{\theta}\left(t_{i j}^{s} v_{i, j}^{s}+t_{i j}^{f} v_{i, j}^{f}\right)+\frac{1}{\theta^{2}}\left(q_{i}^{s}+q_{i}^{f}\right) \theta, i \\
\quad-\frac{1}{\theta} \pi_{i}\left(v_{i}{ }^{f}-v_{i} s\right) \geqq 0
\end{gathered}
$$

Following two inequalities are sufficient conditions to satisfy the inequality (23) in the case of neglecting the body force.

$$
\begin{aligned}
& \theta \bar{\rho}^{s} \frac{d \eta^{s}}{d t}-\bar{\rho}^{s} \frac{d \varepsilon^{s}}{d t}+t_{i j}^{s} v_{i, j}^{s}+\frac{1}{\theta} q_{i}{ }^{s} \theta_{, i}+\theta \bar{\rho}^{f} \frac{d \eta^{f}}{d t} \\
& -\bar{\rho}^{f} \frac{d \varepsilon^{f}}{d t}+t_{i j}^{f} v_{i, j}^{f}+\frac{1}{\theta} q_{i}^{f} \theta, i \geqq 0 \\
& -\pi_{i}\left(v_{i}{ }^{s}-v_{i}{ }^{s}\right) \geqq 0
\end{aligned}
$$

We can take the Eq. (26) as the sufficient condition to satisfy the inequality (25).

$$
\pi_{i}=-d\left(v_{i}^{f}-v_{i}{ }^{s}\right) \quad(d \geqq 0)
$$

More general form of Eq. (26) is

$$
\begin{gathered}
\pi_{i}=-d^{0}\left(v_{i}{ }^{f}-v_{i}^{s}\right)-d^{1}\left(v_{i}{ }^{f}-v_{i}\right)^{3} \cdots \cdots \\
\left(d^{0}, d^{1}, \cdots \cdots \geqq 0\right)
\end{gathered}
$$

From Eq. (26) or (27), it is clear that $\pi_{i}$ is invariant under superposed rigid motion.

\subsection{Equation of Consolidation}

With the aid of Eqs. (13) and (26), Eq. (15) becomes

$$
\frac{\partial n u}{\partial x_{j}} \delta_{i j}=\bar{\rho}^{f} \frac{d v_{i}{ }^{f}}{d t}+d\left(v_{i}{ }^{f}-v_{i} s\right)-\bar{\rho}^{f} b_{i}{ }^{f}
$$

If we can neglect the body force and the acceleration under the condition that $n$ is constant, Eq. (28) is reduced to the Eq. (29).

$$
\frac{\partial u}{\partial x_{i}}=\frac{1}{n} d\left(v_{i}{ }^{f}-v_{i}{ }^{s}\right)
$$

Furthermore, if $\rho^{f}$ and $\rho^{s}$ are constant, Eq. (30) is obtained from Eqs. (5) and (6).

$$
\left[n\left(v_{i} s-v_{i}^{f}\right)\right], i=v_{i, i}^{s}
$$

The coefficient $d$ in Eq. (22) is defined by

$$
d=\rho^{f} g n^{2} / k^{32)}
$$

By Eq. (31), Eq. (29) becomes

$$
\frac{1}{\rho^{f} g} \frac{\partial u}{\partial x_{i}}=\frac{1}{k} n\left(v_{i}^{f}-v_{i}^{s}\right)
$$

where $k$ is the permeability coefficient and $\rho^{f} g$ is the weight of water per unit volume.

As in Eq. (32), $n\left(v_{i}{ }^{f}-v_{i}{ }^{s}\right)$ is considered to be the water influx through the unit surface per unit time, Eq. (32) is reduced to the one-dimensional from Eq. (33) which is equal to the Darcy's law.

$$
v_{i}=k \cdot i \quad i=\frac{\partial u}{\partial x_{i}} \frac{1}{\rho^{f} g}
$$

Differentiating the both side of Eq. (32), under the condition that $n$ is constant, and using the Eqs. (30) and (31), Eq. (34) is obtained.

$$
\frac{1}{\rho^{\jmath} g} \frac{\partial^{2} u}{\partial x_{i}{ }^{2}}=\frac{1}{k} n\left(v_{i, i}^{f}-v_{i, i}^{s}\right)=-\frac{1}{k} v_{i, i}^{s}=-\frac{1}{k} \frac{d \varepsilon_{k k}^{s}}{d t}
$$

Eq. (34) and the stress-strain relation of solid phase form the governing equations of a consolidation.

\subsection{Effective stress concept}

In Eq. (20),

$$
t_{i j}^{s} v_{i}^{s}, j=\left[t_{i j}^{e}+(1-n) u \delta_{i j}\right] v_{i, i}^{s}=t_{i j}^{e} v_{i, i}^{s}+(1-n) u v_{i, i}^{s}
$$

Under the undrained conditions, $v_{i, i}^{s}=v_{i, i}^{f}$ such that $\dot{\varepsilon}_{i i}^{s}=\dot{\varepsilon}_{i i}^{f}$. If fluid is incompressible, $\dot{\varepsilon}_{i i}^{s}=0$. So, $t_{i j}^{(s)} \dot{\varepsilon}_{i j}^{s}$ $=t_{i j}^{e} \dot{\varepsilon}_{i j}^{s} . \quad \dot{\varepsilon}_{i j}^{(\alpha)}$ is the strain rate tensor. From the above discussion, the only stress power reduced by effective stress contribute to the entropy production if fluid is incompressible. In this case, $(1-n) u \delta_{i j}$ is acting on the surface of the solid, but does not contribute to net work. That is to say, if the fluid is nearly incompressible, it is the cause of internal constraint. But, in the 
case of an unsaturated soil, the fluid is fairly compressible. Therefore, $t_{i j}^{e}$ is not effective for the macroscopic deformations. Effective stress is the stress which takes away the stress $(1-n) u \delta_{i j}$ constrained by the fluid from the bulk surface area averaged solid stress $t_{i j}^{s}$. Akai \& Tamura ${ }^{24)}$ determined the pore pressure by adding the constraining condition to the balance equation in their numerical study of multi-dimensional consolidation problem. That is, bulk surface area averaged fluid stress $u n \delta_{i j}$ restrains the volumetric deformation of solid. But, if the stress tensor $(1-n) u \delta_{i j}$ does not exist, the skeleton constituted by soil particles goes to pieces. So, $(1-n) u \delta_{i j}$ can be called the self-support stress tensor. The effective stress causes the macroscopic deformation of saturated soil. Nobody has been clarified the structure of Terzaghi's effective stress concept. But the effective stress is useful in the practical domain of soil engineering. Skempton ${ }^{25}$ challenged the generalization of Terzaghi's effective stress equation. From his original observation, he has shown that Terzaghi's effective stress is not the true effective stress, but excellent approximation for the saturated soils. Three definitions of effective stress were proposed by him.

(a) $\sigma^{\prime}=\sigma-\left(1-a_{c}\right) u$

(b) $\sigma^{\prime}=\sigma-u$

(c) For shear strength,

$$
\sigma^{\prime}=\sigma-\left(1-\frac{a_{c} \tan \phi}{\tan \phi^{\prime}}\right) u
$$

For volume change,

$$
\sigma^{\prime}=\sigma-\left(1-\frac{C_{s}}{C}\right) u
$$

Where $\phi^{\prime}$ is the effective stress angle of shearing resistance $\psi$ the intrinsic friction angle, $C_{s}$ compressibility of soil particle, $C$ soil compressibility, $a_{c}$ contact surface area, $\sigma$ total stress and $\sigma^{\prime}$ effective stress.

These equations were examined in order to see which were able to control the soil behavior. Skempton concluded that Eq. (36) was not a valid representation of effective stress. The Eqs. (38) and (39) account well for shear strength and the volume change of soils, concrete and rock. Eq. (37) is valid only for soils. The above discussion depends on the value of $C, C_{s}, a_{c}$ and $\tan \psi / \tan \phi^{\prime}$. Skempton's generalization of effective stress has following defects.

(1) The definition of a effective stress for shear strength is different from that for compression.
(2) The empirical parameters, which are introduced in the reduction of effective stress in order to connect the intergranular force with the external force, have not the physical basis.

(3) Skempton's approach depends on the assumption that Coulomb's strength equation is valid a priori.

Almost all considerations of the effective stress expressed by many soil engineers, for example, $\mathrm{Lambe}^{26)}$, Scott ${ }^{27)}$ and Mitchell ${ }^{28)}$ et al., follow the Skempton's study. But, from the above observations, Skempton's generalization of the effective stress is too restrictive and not rational. Kenyon $^{29)}$ reduced the self support stress which he called self equilibritated stress in his study of an incompressible solid-fluid mixture. But his theory is restricted to the equilibrium state since he depends on the Müller's mixture theory. The present study is not so. This distinction is due to the difference of the definition of total stress. between the Müller's theory and Green \& Naghdi's. one.

\section{CONSTITUTIVE THEORY FOR A MIX- TURE OF A VISCOELASTIC-VISCOPLAS- TIC MATERIAL AND AN ELASTIC FLUID}

\subsection{Constitutive assumption}

The principle of objectivity requires that the constitutive equation is invariant under the superposed rigid body motion. In order to satisfy this principle, it is sufficient that all tensorial variables are invariant under such motion. Eq. (24) is rewritten by invariant form.

$$
\begin{aligned}
& -\bar{\rho}_{0} s\left(\dot{\psi}^{s}+\eta^{s} \dot{\theta}\right)+T_{K L}^{s} \dot{E}_{K L}^{s}+h_{i} \bar{g}_{i} s / \theta \\
& -\quad \bar{\rho}_{0}^{f}\left(\dot{\psi}^{f}+\eta^{f} \dot{\theta}\right)+T_{K L}^{f} \dot{E}_{K L}^{f}+h_{i}{ }^{f} \bar{g}_{i}{ }^{f} / \theta \geqq 0
\end{aligned}
$$

where $\psi^{\alpha}$ is a fee energy density, $T_{K L}^{\alpha}$ second Kirchhoff stress tensor and $\dot{E}_{K^{\prime} L}^{\alpha}$ strain rate tensor in Lagrangian form. ( $\alpha=s$ or $f$ )

If we introduce the complementary energy density $\phi^{\alpha}$,

$$
\phi^{\alpha}=\frac{1}{\bar{\rho}_{0}^{\alpha}} T_{K L}^{\alpha} E_{K L}^{\alpha}-\psi^{\alpha}
$$

Eq. (40) takes the following form.

$$
\begin{gathered}
\bar{\rho}_{0} s \dot{\phi}^{s}-E_{K L}^{s} \dot{T}_{K L}^{s}-\bar{\rho}_{0}^{s} \dot{\theta} \eta^{s}+h_{i}{ }^{s} \bar{g}_{i} s / \theta+\bar{\rho}_{0}{ }^{j} \dot{\phi}^{f} \\
\quad-E_{K L}^{f} \dot{T}_{K L}^{f}-\bar{\rho}_{0} \dot{f} \dot{\theta} \eta^{f}+h_{i}{ }^{f} \bar{g}_{i}{ }^{f} / \theta \geqq 0 \ldots \ldots \ldots \ldots
\end{gathered}
$$

where $h_{i}{ }^{\alpha}=\frac{\bar{\rho}_{0}{ }^{\alpha}}{\bar{\rho}^{\alpha}} F_{i j}^{-1 \alpha} q_{j}{ }^{\alpha}, \quad \bar{g}_{i}{ }^{\alpha}=F_{i j}^{T \alpha} \theta, j$ and $F_{i j}^{\alpha}$ is: a deformation gradient tensor. Principle of equi-presence is a rule for a mathematical convenience, but not a physical principle. So, this. principle need not be satisfied always. The 
behavior of the solid phase is characterized by eleven response functions.

$$
\begin{aligned}
& \phi^{s}=\hat{\phi}_{1}^{s}\left(E_{K L}^{v p}, T_{K L}^{s}, \kappa, \theta, \bar{g}_{I} s\right) \\
& +\hat{\phi}_{2} s\left(E_{K L}^{v e}, T_{K L}^{e}, \theta, \bar{g}_{I}^{s}\right) \\
& \phi^{f}=\hat{\phi}^{f}\left(T_{K K}^{f}, \theta, \bar{g}_{1}^{f}\right) \\
& \eta^{s}=\hat{\eta}^{s}\left(E_{K L}^{v p}, E_{K L}^{r e}, T_{K L}^{s}, \kappa, \theta, \bar{g}_{I}^{s}\right) \\
& \eta^{f}=\hat{\eta}^{f}\left(T_{K K}^{f}, \theta, \bar{g}_{I}^{f}\right) \\
& E_{K L}^{s}=\hat{E}_{K L}^{s}\left(E_{K L}^{v p}, E_{K L}^{v e}, T_{K L}^{s}, \kappa, \theta, \bar{g}_{I}^{s}\right) \\
& E_{K K}^{f}=\hat{E}_{K K}^{f}\left(T_{K K}^{f}, \theta, \bar{g}_{I} f\right) \\
& h_{i} s=\hat{h}_{i}{ }^{s}\left(E_{K L}^{v p}, E_{K L}^{v e}, T_{K L}^{s}, \kappa, \theta, \bar{g}_{I} s\right) \\
& h_{i}^{f}=\hat{h}_{i}{ }^{f}\left(T_{K K}^{f}, \theta, \bar{g}_{I}{ }^{f}\right) \\
& \dot{E}_{K L}^{v p}=\hat{\dot{E}}_{K L}^{v p}\left(E_{K L}^{v p}, T_{K L}^{s}, \kappa, \theta, \bar{g}_{I} s\right) \\
& \dot{E}_{K L}^{v e}=\hat{\dot{E}}_{K L}^{v e}\left(E_{K L}^{v e}, T_{K L}^{s}, \theta, \bar{g}_{I} s\right) \cdots \\
& \dot{\kappa}=\hat{\dot{\kappa}}\left(E_{K L}^{v p}, T_{K L}^{s}, \kappa, \theta, \bar{g}_{I}^{s}\right)
\end{aligned}
$$

$E_{K L}^{v p}$ and $E_{K L}^{v e}$ are the internal state variables corresponding to viscoplastic strain and viscoelastic strain respectively. $\kappa$ is the scalar parameter which is an internal variable for hardening parameter. Eqs. (50), (51) and (52) are evolution equations that govern the internal variables. In Eqs. (42) (52), it is assumed that the coupling between solid and fluid does not exist. The case that such coupling exist is discussed later. The rate equations of $\phi^{s}$ and $\phi^{f}$ are

$$
\begin{aligned}
\dot{\phi}^{s}= & \frac{\partial \phi^{s}}{\partial T_{K L}} \dot{T}_{K L}^{s}+\frac{\partial \phi}{\partial E_{K L}^{v p}} \dot{E}_{K L}^{v p}+\frac{\partial \dot{\phi}_{1}^{s}}{\partial \kappa} \dot{\kappa} \\
& +\frac{\partial \phi_{2}^{s}}{\partial E_{K L}^{v e}} \dot{E}_{K L}^{v e}+\frac{\partial \phi}{\partial \theta} \dot{\theta}+\frac{\partial \phi^{s}}{\partial \bar{g}_{i}^{s}} \bar{g}_{i}^{s} \\
\dot{\phi}^{f}= & \frac{\partial \phi^{f}}{\partial T_{K K}^{f}} \dot{T}_{K K}^{f}+\frac{\partial \phi^{f}}{\partial \theta} \dot{\theta}+\frac{\partial \phi^{f}}{\partial \bar{g}_{i}^{f}} \dot{\bar{g}}_{i}{ }^{f}
\end{aligned}
$$

Substituting Eq. (53) into Eq. (41),

$$
\begin{aligned}
& \left(\frac{\partial \phi^{s}}{\partial T_{K L}^{s}}-\frac{1}{\bar{\rho}_{0}^{s}} \dot{E}_{K L}^{s}\right) \dot{T}_{K L}^{s}+\frac{\partial \phi_{1}^{s}}{\partial E_{K L}^{v p}} \dot{E}_{K L}^{v p}+\frac{\partial \dot{\phi}_{2}^{s}}{\partial E_{K L}^{v e}} \dot{E}_{K L}^{v e} \\
& +\frac{\partial \phi_{1}^{s}}{\partial \kappa} \kappa+\left[\frac{\partial\left(\phi_{1}^{s}+\phi_{1}^{f}\right)}{\partial \theta}-\eta^{f}-\eta^{s}\right] \dot{\theta} \\
& +\frac{\partial \phi^{f}}{\partial \bar{g}_{I}^{f}} \dot{\bar{g}}_{i}^{f}+\frac{\partial \phi^{s}}{\partial \bar{g}_{I} s} \dot{\bar{g}}_{i} s+\left(\frac{\partial \phi^{f}}{\partial T_{K K}^{f}}-\frac{1}{\bar{\rho}_{0}^{f}} E_{K K}^{f}\right) \dot{T}_{K K}^{f} \\
& +\left(\bar{h}_{I} s \bar{g}_{I} s+\bar{h}_{I} s \bar{g}_{I}^{f}\right) / \theta \geqq 0
\end{aligned}
$$

Following the Coleman's method, ${ }^{4)}$ we can conclude that

$$
\begin{aligned}
& \eta^{f}+\eta^{s}=\frac{\partial\left(\phi^{f}+\phi^{s}\right)}{\partial \theta} \\
& E_{K L}^{s}=\bar{\rho}_{0} s \frac{\partial \phi^{s}}{\partial T_{K L}^{s}} \\
& E_{K K}^{f}=\bar{\rho}_{0} \mathcal{f} \frac{\partial \phi^{f}}{\partial T_{K K}^{f}} \\
& \frac{\partial \phi^{f}}{\partial \bar{g}_{i}^{f}}=0
\end{aligned}
$$

$$
\begin{aligned}
& \frac{\partial \phi^{s}}{\partial \bar{g}_{i}^{s}}=0 \\
& \left(h_{i}{ }^{\mathcal{g}} \bar{g}_{i}{ }^{\mathcal{f}}+h_{i}{ }^{s} \bar{g}_{i}{ }^{s}\right) / \theta \geqq 0 \\
& \frac{\partial \phi_{1}^{s}}{\partial E_{K L}^{v p}} \dot{E}_{K L}^{v p}+\frac{\partial \phi_{2}^{s}}{\partial E_{K L}^{v e}} \dot{E}_{K L}^{v e}+\frac{\partial \dot{\phi}_{1}^{s}}{\partial \kappa} \dot{\kappa} \geqq 0
\end{aligned}
$$

Since the energy dissipation is attributed not only to plastic work but also to viscoplastic work, the inequality (61) will be divided into two parts, each of which is therefore assumed to be positive or zero. Resultantly, these inequalities (62) and (63) corresponds to a sufficient condition of Eq. (61).

$$
\begin{aligned}
& \dot{E}_{K L}^{v p} \frac{\partial \phi_{1}^{s}}{\partial E_{K L}^{v p}}+\dot{\kappa} \frac{\partial \phi_{1}^{s}}{\partial \kappa} \geqq 0 \\
& \dot{E}_{K L}^{v e} \frac{\partial \phi_{1}^{s}}{\partial E_{K L}^{v e}} \geqq 0 \quad \ldots \ldots \ldots . . .
\end{aligned}
$$

We also postulate Eqs. (64), (65) and (66).

$$
\begin{aligned}
& \dot{E}_{K L}^{v p}=M_{K L I J} \frac{\partial \phi_{1} s}{\partial E_{I J}^{v p}} \\
& \dot{\kappa}=G_{K L} \dot{E}_{K L}^{v p} \\
& \cdots \cdots \cdots \cdots \\
& \dot{E}_{K L}^{v e}=\bar{\eta}_{K L I J} \frac{\partial \phi_{2}{ }^{s}}{\partial E_{I J}^{v e}}
\end{aligned}
$$

Eq. (64) shows that $\dot{E}_{K L}^{v p}$ is not generally normal to the complementary energy function $\phi_{1}{ }^{s}$. Eq. (65) indicates that $\dot{\kappa}$ is a function of only the rate of viscoplastic strain. In this sense, $\kappa$ corresponds to the strain-hardening parameter used in the classical theory of plasticity.

Complementary energy densities are assumed as follows for future use.

$$
\begin{aligned}
\bar{\rho}_{0}{ }^{s} \phi^{s}= & E_{K L}^{v p} T_{K L}^{s}+E_{K L}^{v e} T_{K L}^{s} \\
& -\gamma_{I J K L}^{1} E_{I J}^{v e} E_{K L}^{v e}+G\left(T_{I J}^{s}\right) \ldots \ldots \ldots \\
\gamma_{I J K L}^{1}= & a^{1} \delta_{I J} \delta_{K L}+b^{1}\left(\delta_{I K} \delta_{J L}+\delta_{I L} \delta_{J K}\right) \\
\bar{\rho}_{0}{ }^{f} \phi^{f}= & \left(T_{K K}^{f}\right)^{2} m_{J} / 6 \quad \ldots \ldots \ldots \ldots \ldots \ldots \ldots \ldots \ldots \ldots \ldots \ldots \ldots \ldots \ldots \ldots
\end{aligned}
$$

where, $a^{1}, b^{1}$ and $m_{f}$ are material constants.

From Eqs. (56) and (67),

$$
E_{K L}^{s}=E_{K L}^{v p}+E_{K L}^{v e}+\frac{\partial G}{\partial T_{K L}^{s}}
$$

Similarly, from Eqs. (57) and (68), Eq. (70) is given.

$$
E_{K K}^{f}=\text { num }_{f}
$$

where $u$ is a pore water pressure and $m_{f}$ is an intrinsic compressibility of a fluid. Usually, $m_{f}$ is smaller than the compressibility of soil skeleton for saturated soil. So, in the following, $m_{f}$ is assumed to be zero. That is to say, fluid is incompressible. In the case that a pore fluid is incompressible, from the discussion in section 3.4 , we must use the effective stress tensor $T_{I J}^{e}$ in place of the bulk area averaged solid stress 
tensor $T_{I J}^{s}$. The viscoplastic strain which is Viogt type is introduced by taking Eq. (71) as the explicit description of Eq. (66).

$$
E_{K L}^{v e}=\bar{\eta}_{K L M N}\left(T_{M N}^{e}-\gamma_{M N I J}^{1} E_{I J}^{v e}\right)
$$

If $\bar{\eta}_{K L M N}$ is a fourth order isotropic tensor,

$$
\bar{\eta}_{K L M N}=a^{2} \delta_{K L} \delta_{M N}+b^{2}\left(\delta_{K M} \delta_{L N}+\delta_{K N} \delta_{L M}\right)
$$

Eq. (71) becomes

$$
\begin{aligned}
& \dot{E}_{K L}^{v e}=3 a^{2} T_{m}^{e} \delta_{K L}+2 b^{2} s_{K L}-3 \tau^{1} E_{K L}^{v e}-2 \tau^{2} e_{K L}^{v e} \\
& \ldots \ldots \ldots \ldots \ldots \ldots \ldots \ldots \ldots \ldots \ldots \ldots \ldots \ldots \ldots \\
& \tau^{1}=3 a^{1} a^{2}+2 a^{1} b^{2}+2 b^{1} a^{2} \\
& \tau^{2}=2 b^{1} b^{2} \\
& e_{K L}=E_{K L}-\frac{1}{3} E_{M M} \delta_{K L} \\
& T_{m}^{e}=T_{K K}^{e} / 3, \quad s_{K L}=T_{K L}^{e}-T_{m}^{e} \delta_{K L}
\end{aligned}
$$

Finally, Eq. (69) is reduced to

$$
\begin{aligned}
\dot{E}_{K L}^{s}= & \dot{E}_{K L}^{v p}+\left(\overline{\frac{\dot{\partial G}}{\partial T_{K L}^{e}}}\right)+\left(3 a^{2} T_{m}^{e}-3 \tau^{1} E_{M M}^{v e}\right) \delta_{K L} \\
& +\left(2 b^{2} s_{K L}-2 \tau^{2} e_{K L}^{v e}\right) \cdots \cdots \cdots \cdots \cdots \cdots \cdots \cdots \cdots \cdots \cdots \cdots \cdots \cdots
\end{aligned}
$$

The function $f$ is defined by

$$
f \operatorname{def} \int_{0}^{T_{I J}} A_{I J} d T_{I J} \quad\left(A_{I J}=N_{I J K L} \frac{\partial \phi_{1}^{s}}{\partial E_{K L}^{v p}}\right)
$$

Integration is carried under the condition that $E_{I J}^{v p}$ is constant. This definition is more general than in the author's previous paper. ${ }^{12)}$

$$
\text { If } \begin{aligned}
\frac{\partial A_{I J}}{\partial T_{K L}^{e}} & =\frac{\partial A_{K L}}{\partial T_{I J}^{e}}, \\
A_{I J} & =\frac{\partial f}{\partial T_{I J}^{e}}
\end{aligned}
$$

From Eq. (64),

$$
\dot{E}_{I J}^{v p}=M_{I J K L}\left(N_{K L M N}\right)^{-1} \frac{\partial f}{\partial T_{M N}^{e}}
$$

where $M_{I J K L}\left(N_{K L M N}\right)^{-1} \frac{\partial f}{\partial T_{M N}^{e}}=\frac{\partial f}{\partial T_{I J}^{e}}$.

Complementary energy $\phi$ of viscoelastic-viscoplastic body is illustrated in Fig. 5.

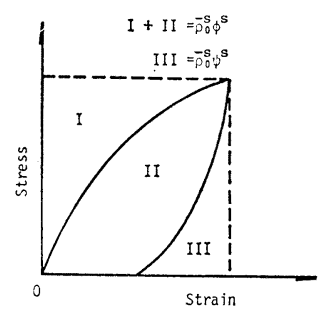

Fig. 5 Free energy $\psi$ and Complementary energy $\phi$ for Viscoelastic-Viscoplastic body.
When the soil particle and the fluid are compressible, following relations must be given if the coupling between solid and fluid influences the volumetric strain only.

$$
\left.\begin{array}{l}
E_{K K}^{s}=E_{K K}^{s}\left(T_{I J}^{f}, T_{I J}^{s}, E_{I J}^{v p}, E_{I J}^{v e}, \theta, \bar{g}_{I}^{s}, \kappa\right) \\
E_{K K}^{f}=E_{K K}^{f}\left(T_{I J}^{f}, T_{I J}^{s}, E_{I J}^{v p}, E_{I J}^{v e}, \theta, \bar{g}_{I}^{s}, \kappa\right)
\end{array}\right\}
$$

The deformation of a solid phase depends upon that of a fluid. For example, Biot takes the linear relation Eq. (79) as the Eq. (78).

$$
\left.\begin{array}{l}
E_{K K}^{s}=\frac{1}{\left(k_{c} \alpha_{b}-\alpha_{c}\right)}\left\{\left[(1-n) u+T_{m}^{e}\right] k_{c}-n u \alpha_{c}\right\} \\
E_{K K}^{f}=\frac{1}{\left(k_{c} \alpha_{b}-\alpha_{c}\right)}\left\{n u \alpha_{b}-\left[(1-n) u+T_{m}^{e}\right] \alpha_{c}\right\}
\end{array}\right\}
$$

where $\alpha_{b}, \alpha_{c}$ and $k_{c}$ are material constants. ${ }^{32}$ The case that the internal constrain is a special case as two phase mixture and the essence of the mixture appears only when the coupling between two phases like Eq. (78) exists. There must exist a relative velocity between solid and fluid phase and this term should be taken as an independent variable in derivation of the constitutive equation. From a thermodynamic restriction, ${ }^{30)}$ and/or a property of wave phenomena, $\left.{ }^{31}\right)$ however, the first order term of relative velocity vanishes deductively under a condition with a special assumption. Moreover, higher order terms of the relative velocity than the second order is neglected from the consideration.

\subsection{Constitutive theory for a normally consolidat- ed clay}

We will be limited to the infinitesimal strain field and isothermal condition. Adachi \& Okano ${ }^{32)}$ extended the Roscoe's original theory to the three dimensional case. The extended static yield function is given by

$$
f_{s}=\sqrt{2 J_{2}}+M^{*} \sigma_{m}^{\prime} \ln \left(\sigma_{m}^{\prime} / \sigma_{m y}^{\prime}\right) \cdots
$$

where $\quad 2 J_{2}=s_{i j} s_{i j}, \quad s_{i j}=\sigma^{\prime}{ }_{i j}-1 / 3 \sigma^{\prime}{ }_{k k} \delta_{i j}, \quad \sigma_{m}^{\prime}=$ $1 / 3 \sigma_{k k}^{\prime}, \sigma^{\prime}{ }_{i j}$ is an effective stress tensor and $M^{*}$ is the value of $\sqrt{2 J_{2}} / \sigma_{m}^{\prime}$ at critical state. and $\sigma_{m y}^{\prime}$ is a hardening parameter. The yield condition of Von Mieses asserts that the material yields and flow plastically when the elastic energy reaches some critical quantity.

$$
f=\frac{1}{2} s_{i j s i j}-\kappa=0 \text {. }
$$

The yield condition Eq. (80) is regarded as the extension of that of Von Mises. So, Eq. (80) is replaced by Eq. (82) corresponding to Eq. (81). 


$$
f_{s}=2 J_{2}-\left(M^{*} \sigma_{m}^{\prime} \ln \left(\sigma_{m}^{\prime} / \sigma_{m y}^{\prime}\right)\right)^{2}
$$

The reason that $f_{s}$ is given by Eq. (82) is as follows. Function $f$ must be connected with the energy since $f$ is defined by Eq. (74). If the yield condition $f_{s}=0$ is used, the results obtained by using Eq. (82) is the same as that by using Eq. (80) as plastic potential. $N_{i j k l}$ and $M_{i j k l}$ are postulated as isotropic tensor.

$$
\begin{aligned}
N_{i j k l}= & A \delta_{i j} \delta_{k l}+B\left(\delta_{i k} \delta_{j l}+\delta_{i l} \delta_{j k}\right) \cdots \cdots \cdots \cdots . . .(83) \\
A= & \frac{1}{3}\left[-\frac{2 M^{*}}{3 \sigma_{m}^{\prime}}\left(M^{*} \sigma_{m}^{\prime} \ln \left(\sigma_{m}^{\prime} / \sigma_{m y}^{\prime}\right)-F\right)\right. \\
& \left.\cdot\left(\ln \left(\sigma_{m}^{\prime} / \sigma_{m y}^{\prime}\right)+1\right)-2\right], \quad B=1 \\
M_{i j k l}= & P \delta_{i j} \delta_{k l}+Q\left(\delta_{i k} \delta_{j l}+\delta_{i l} \delta_{j k}\right) \cdots \cdots \cdots \cdots \cdots(84) \\
P= & (3 A+2) X+2 A Y, \quad Q=2 Y \\
X= & \left(\beta^{2}-\beta^{1}\right) / 6 \sqrt{2 J_{2}}, \quad Y=\beta^{1} / 4 \sqrt{2 J_{2}}
\end{aligned}
$$

From Eqs. (74) and (83),

$$
\begin{aligned}
& A_{i j}=2 s_{i j}-\frac{2}{3} M^{*}\left(M^{*} \sigma_{m}^{\prime} \ln \left(\sigma_{m}^{\prime} / \sigma_{m y}^{\prime}\right)-F\right) \\
& \cdot\left(\ln \left(\sigma_{m}^{\prime} / \sigma_{m y}^{\prime}\right)+1\right) \delta_{i j}
\end{aligned}
$$

Therefore, $\frac{\partial A_{i j}}{\partial \sigma_{m n}^{\prime}}=\frac{\partial A_{m n}}{\partial \sigma_{i j}^{\prime}}$.

Then $f$ is $f=2 J_{2}-\left(M^{*} \sigma_{m} \ln \left(\sigma_{m}^{\prime} / /_{m y}^{\prime}\right)-F\right)^{2}$.

From Eq. (64),

$$
\dot{\varepsilon}_{i j}^{v p}=M_{i j k l} \frac{\partial \dot{\phi}_{1}{ }^{s}}{\partial \varepsilon_{k l}^{v p}}=(3 P+2 Q) \sigma_{m}^{\prime} \delta_{i j}+2 Q s_{i j} \cdots
$$

Substituting Eq. (86) into Eq. (62), using $\partial \phi^{s} / \partial \kappa=$ 0 , we get

$$
\begin{aligned}
\dot{\varepsilon}_{i j}^{v p} \frac{\partial \dot{\phi}_{1}^{s}}{\partial \varepsilon_{i j}^{v p}} & =\beta^{2} M^{*}\left(\ln \left(\sigma_{m}^{\prime} / \sigma_{m y}^{\prime}\right)+1\right) \sigma_{m}^{\prime}+\sqrt{2 J_{2}} \beta^{1} \\
& =F \beta^{2}+\sqrt{2 J_{2}}\left(\beta^{1}-\beta^{2}\right)+\sigma_{m}^{\prime} M^{*} \beta_{2} \cdots(87)
\end{aligned}
$$

If $\beta^{1}$ and $\beta^{2}$ are positive functions, Eq. (88) is a sufficient condition because $F$ is positive.

$$
\beta^{1}-\beta^{2} \geqq 0
$$

From the Eqs. (73), (77), (83) and (84), the stressstrain relation for a normally consolidated clay is obtained.

$$
\begin{aligned}
\dot{\varepsilon}_{i j}= & \gamma_{1} \dot{s}_{i j}+\frac{2}{3} \gamma_{2} \dot{\sigma}_{m}^{\prime} \delta_{i j}+\left(2 b^{2} s_{i j}-2 \tau^{2} e_{i j}^{v e}\right) \\
& +\left(3 a^{2} \sigma_{m}^{\prime}-3 \tau^{1} \varepsilon_{k k}^{v e}\right) \delta_{i j}+\frac{s_{i j}}{\sqrt{2 J_{2}}} \beta^{1} \\
& +\frac{1}{3} \delta_{i j} \beta^{2}\left\{M^{*}-\frac{\sqrt{2 J_{2}^{s}}}{\sigma_{m}^{\prime s}}+M^{*} \ln \left(\sigma_{m}^{\prime} / \sigma_{m}^{\prime s}\right)\right\}
\end{aligned}
$$

\section{ONE-DIMENSIONAL STRESS WAVE PRO- PAGATION THROUGH COHESIVE SOIL}

\subsection{Wave equation}

Bar wave propagation can be observed under the condition that the lateral displacement is not confined and wave length is very small than the diameter of the bar. So, boundary condition is given by stress condition. We observed the wave of this type at the stress wave propagation test $t^{33}$ using the shock tube. Stress condition under the triaxial compression test is as follows.

$$
\sigma_{i j}=\left[\begin{array}{lll}
\sigma_{11} & 0 & 0 \\
0 & \sigma_{22} & 0 \\
0 & 0 & \sigma_{33}
\end{array}\right], \quad \sigma_{22}=\sigma_{33}
$$

As $\sigma_{33}$ is constant under the undrained condition, the equation of motion in one-dimensional case is

$$
\begin{aligned}
& \frac{\partial\left(\sigma_{11}^{s}-\sigma_{33}^{s}-\sigma_{11}^{f}\right)}{\partial x}+\frac{\partial \sigma_{11}^{f}}{\partial x}=\bar{\rho}_{0}^{f} \frac{d v_{1}^{f}}{d t}+\bar{\rho}_{0}^{s} \frac{d v_{1}^{s}}{d t} \\
& \sigma_{11}^{f}=n u
\end{aligned}
$$

after all,

$$
\frac{\partial q}{\partial x}=\bar{\rho}_{0}^{s} \frac{d v_{1}^{s}}{d t}+\bar{\rho}_{0}^{f} \frac{d v^{f}}{d t} \quad q=\sigma_{11}^{\prime}-\sigma_{33}^{\prime}
$$

The equation of motion for several constituents are denoted by Eqs. (91) and (92).

$$
\begin{aligned}
& \frac{\partial q}{\partial x}=\bar{\rho}_{0}^{s} \frac{d v_{1}^{s}}{d t}+\frac{\partial(n u)}{\partial x}+\pi_{1} \\
& \frac{\partial(n u)}{\partial x}=\bar{\rho}_{0}^{f} \frac{d v^{f}}{d t}-\pi_{1} \cdots \ldots \ldots . . \\
& \pi_{1}=-d\left(v_{1}^{f}-v_{1}^{s}\right)
\end{aligned}
$$

If $v_{1}^{s}$ is equal to $v_{1}{ }^{f}$, equation of motion becomes

$$
\frac{\partial q}{\partial x}=\rho_{0} \frac{\partial v^{s}}{\partial t} \quad\left(\rho_{0}=\bar{\rho}_{0}^{s}+\bar{\rho}_{0}^{f}\right)
$$

According to Ishihara ${ }^{34)}$, if $v_{1}^{f}$ is not equal to $v_{1}^{s}$ and frequency range is from $1 \mathrm{cps}$ to $30 \mathrm{cps}$, interaction term $\pi_{1}$ is predominant rather than inertia term $\bar{\rho}_{0} f \frac{d v^{f}}{d t}$. So, the form of the motion comes to consolidation. Therefore, in the ordinary range of frequency, a motion of soil becomes wave only if $v_{i}^{s}$ equals to $v_{i}{ }^{f}$. The pulse in the stress wave propagation tests carried by authors has the several hundred frequency. If the water is incompressible and cohesive soil is modeled by a elastic-viscoplastic body reported in the previous paperis),

$$
\begin{aligned}
& \left|d\left(v_{1}^{f}-v_{1}^{s}\right)\right|=1.48 \times 10^{3}\left(\mathrm{~kg} / \mathrm{m} \mathrm{sec}^{4}\right), \\
& \bar{\rho}_{0}^{f} \frac{d v_{1}^{f}}{d t}=1.19 \times 10^{4}\left(\mathrm{~kg} / \mathrm{m} \mathrm{sec}^{4}\right), \\
& \bar{\rho}^{s} \frac{d u^{s}}{d f}=3.71 \times 10^{4}\left(\mathrm{~kg} / \mathrm{m} \mathrm{sec}^{4}\right)
\end{aligned}
$$

At this time, permeability coefficient $k$ is $2.14 \times$ $10^{-4} \mathrm{~cm} / \mathrm{sec}$. The stress-strain relation used for calculation is as follows.

$$
\dot{\varepsilon}_{11}=\frac{1}{E} \dot{q}+C_{1} \exp \left[\frac{m}{\sigma_{m e}^{\prime}}\left(q-q^{s}\right)\right]
$$




$$
\begin{aligned}
& \dot{\sigma}_{m}^{\prime}=-\frac{C_{2}}{\gamma_{2}} \exp \left[\frac{m}{\sigma_{m e}^{\prime}}\left(q-q^{s}\right)\right] \\
& C_{1}=10^{-4}(1 / \mathrm{sec}), C_{2} / \gamma_{2}=100\left(\mathrm{~kg} / \mathrm{m}^{2} / \mathrm{sec}\right), m=23,
\end{aligned}
$$

The calculation procedure used here is finite difference method. In this case, interaction term is not necessarily predominant than inertia term. But the attenuation of stress wave is scarcely different between the cases which has several different permeabilities. When $k$ is $2.14 \times$ $10^{-4} \mathrm{~cm} / \mathrm{sec},\left|v_{1}^{f}-v_{1}^{s}\right|$ is about $10^{-5} \mathrm{~m} / \mathrm{sec}$. Taking the above discussion into account, we can postulate that $v_{1}{ }^{f}$ is equal to $v_{1}^{s}$.

\subsection{Stress Wave propagation through saturated cohesive soil}

We shall treat the stress wave propagation through the bar of saturated cohesive soil whose behavior is described by the Eq. (89). From the discussion in section 5.1 , we can postulate that $v_{1}{ }^{f}$ is equal to $v_{1}^{s}$. So, the equation of motion is given by Eq. (93). If we can assume volumetric strain is zero, in one-dimensional case Eq. (89) becomes

$$
\dot{\varepsilon}_{11}=\frac{1}{E} \dot{q}+\left(\frac{4}{3} b^{2} q-2 \tau^{2} e_{11}^{v e}\right)+\sqrt{\frac{2}{3}} \beta_{1}(F)
$$

Furthermore, if viscoelastic volume strain is zero,

$$
2 \gamma_{2} \dot{\sigma}_{m}^{\prime}+\beta_{2}\left[M^{*}-\frac{\sqrt{2 J_{2}^{s}}}{\sigma_{m}^{\prime s}}+M^{*} \ln \left(\sigma_{m}^{\prime} / \sigma_{m}^{\prime s}\right)\right]=0
$$

In the similar manner of previous paper, ${ }^{12)}$

$$
\begin{aligned}
& \sqrt{\frac{2}{3}} \beta_{1}=C_{1} \exp \left[\frac{m}{\sigma_{m e}^{\prime}}\left(q-q^{s}\right)\right] \\
& \beta_{2}=\bar{C}_{1} \exp \left[\frac{m}{\sigma_{m e}^{\prime}}\left(q-q^{s}\right)\right] \ldots \ldots
\end{aligned}
$$

The parameters $m, C_{1}$ and $\bar{C}_{1}$ are considered to depend on the value of strain which can be given by the strain-rate constant triaxial compression test. If $\varepsilon_{11}$ is positive in compression, the relation between strain and particle velocity is given by

$$
-\frac{\partial \varepsilon_{11}}{\partial t}=\frac{\partial v_{1}}{\partial x_{1}}
$$

Eqs. (93), (94) and (98) from quasi-linear partial differential equations. The characteristics are

$$
d x_{1}=0 \text { and } d x_{1} / d t= \pm \sqrt{\frac{E^{\prime}}{\rho_{0}}}= \pm c
$$

Along these characteristics, the following differential relations exist.

$$
\text { Along } d x_{1}=0, d \varepsilon_{11}=\frac{1}{E^{\prime}} d q+\left(\frac{4}{3} b^{2} q-2 \tau^{2} e^{v e}\right) d t
$$

$$
\begin{aligned}
& +\sqrt{\frac{2}{3}} \beta^{1}(F) d t \\
& \text { Along } \frac{d x_{1}}{d t}= \pm \sqrt{\frac{E^{\prime}}{\rho_{0}}}= \pm c, \quad d v_{1}=\mp \frac{1}{\rho_{0} c} d q \\
& -\left[\left(\frac{4}{3} b^{2} q-2 \tau^{2} e_{11}^{v e}\right)+\frac{2}{3} \beta_{1}(F)\right] d x_{1}
\end{aligned}
$$

\subsection{Numerical results and consideration}

Numerical calculation is carried by integrating the ordinary differential relation along the characteristics. The visco elastic parameter $E\left(=3 \tau^{2} /\right.$ $\left.2 b^{2}\right)$ and $\mu\left(=4 / 3 b^{2}\right)$ can be determined by the Akai and Hori's ${ }^{10)}$ viscoelastic approach to soil. Akai and Hori concluded in their research that the physical behavior of soil is viscoelastic in the strain level of $10^{-4}-10^{-3}$ and the soil can be assumed to be described by linear spring-Voigt model in wide frequency range. The viscoelastic parameter $\tilde{k}\left(=E / E^{\prime}\right)$ and relaxation time constant $\tau(=1 / E \mu)$ take the value of $0.1-0.5$ and $10^{-2}-5 \times 10^{-2}(\mathrm{sec})$ respectively. $E^{\prime}$ is the Young's modulus of free spring and $E$ is the elastic modulus of Voigt part in the spring-Voigt model. $1 / \mu$ is the viscosity coefficient. Table 1 shows the fixed parameters in the calculation. The parameters $m$ and $C_{1}$ used in the calculation are as follows.

Table 1 Parameters used in numerical calculation.

\footnotetext{
Young's Modulus $E^{\prime}=1.73 \times 10^{7}\left(\mathrm{~kg} / \mathrm{m}^{2}\right)$

Density $\quad \bar{\rho}_{0}=196.3\left(\mathrm{~kg} / \mathrm{m}^{2} \mathrm{sec}^{2}\right)$

Slope of $e-\log \sigma^{\prime} m$ line of consolidation test $\lambda=0.127$

Slope of $e-\log \sigma^{\prime} m$ line of swelling test $\kappa=0.0214$

Value of $\left(\left(\sigma_{11}^{\prime}-\sigma^{\prime}{ }_{33}\right) / \sigma^{\prime} m\right)$ at critical state $M^{*}=1.300$

Consolidation pressure $\sigma_{m e}^{\prime}=1.06\left(\mathrm{~kg} / \mathrm{cm}^{2}\right)$

Void ratio $e_{0}=0.77$

$k\left(=E / E^{\prime}\right)=0.32, \mu=1.75 \times 10^{-5}\left(1 / \mathrm{sec} / \mathrm{kg} / \mathrm{m}^{2}\right)$

$\bar{C}_{1} / 2 \gamma_{2}=10.0 \times C_{1}\left(\mathrm{~kg} / \mathrm{m}^{2} / \mathrm{sec}\right)$

$$
\begin{aligned}
m= & -1400 \varepsilon_{11}+37.0\left(\varepsilon_{11}<10^{-2}\right) \\
m= & 23.0 \quad\left(\varepsilon_{11}>10^{-2}\right) \\
C_{1}= & {\left[1.8 \times 10^{-14} \times\left(100 \varepsilon_{11}\right)^{3.06}+10^{-17}\right] } \\
& \cdot \exp \left(m q_{s} / \sigma_{m e}^{\prime}\right)\left(\varepsilon_{11}<10^{-2}\right)(1 / \mathrm{sec}) \\
C_{1}= & {\left[1.8 \times 10^{-14}+10^{-17}\right] } \\
& \cdot \exp \left(m q_{s} / \sigma_{m e}^{\prime}\right)\left(\varepsilon_{11}>10^{-2}\right)(1 / \mathrm{sec})
\end{aligned}
$$$$
\bar{k}=0.32\left(E / E^{\prime}\right) \quad \tau=1.03 \times 10^{-2}(\mathrm{sec})
$$

Figs. 6 and 7 shows the wave variation during the wave propagation through the cohesive soil bar. Fig. 7 is the previously reported case that viscoelastic element in the model is neglected. Comparing Fig. 6 with Fig. 7, the attenuation of peak stress in Fig. 7 is larger than that of Fig. 6. But the tendency of peak stress attenuation is
} 


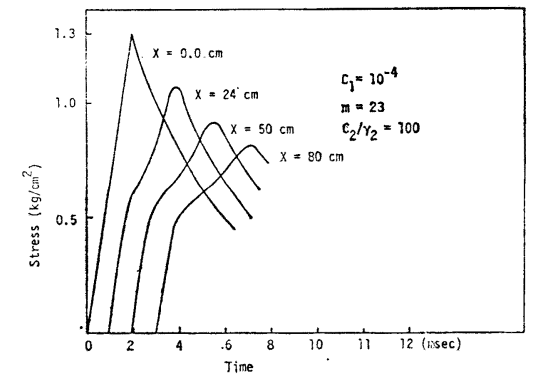

Fig. 6 Stress-Time Relationship (calculated results)

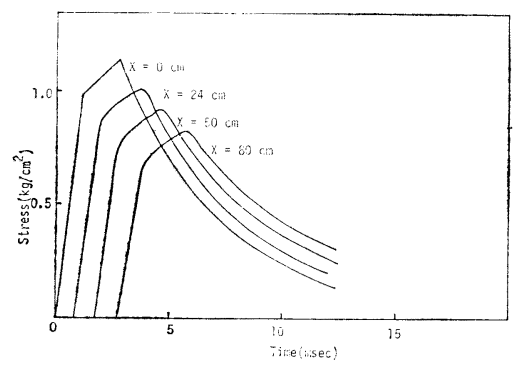

Fig. 7 Stress-Time Relationship (calculated results)

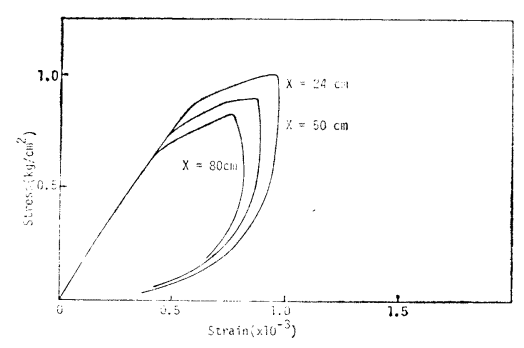

Fig. 8 Stress-Strain Relation (calculated results)

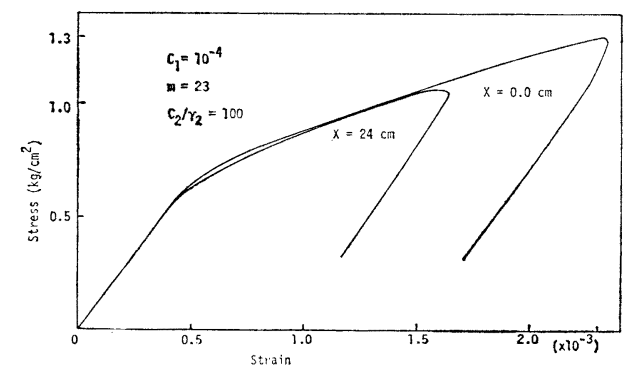

Fig. 9 Stress-Strain Relation (calculated results)

equal between these two cases. The rise time becomes large as the wave propagates in these figures. Figs. 8 and 9 show the stress-strain rela-

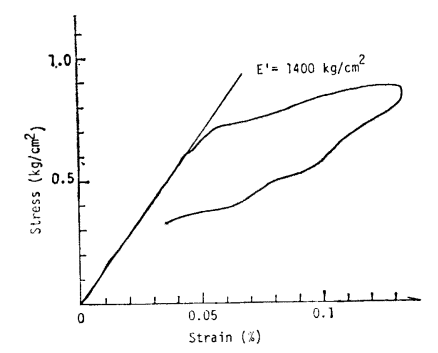

Fig. 10 Stress-Strain Relation During the Wave Propagation Test (after Akai and Hori $^{311}$ )

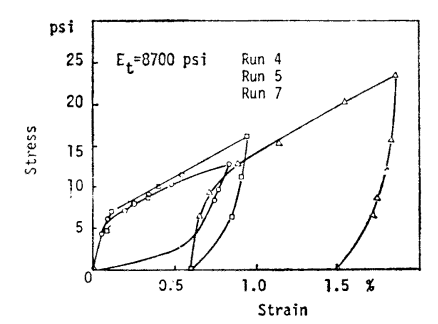

Fig. 11 Dynamic Stress-Strain curves (after Vey \& Strauss $^{32)}$ )

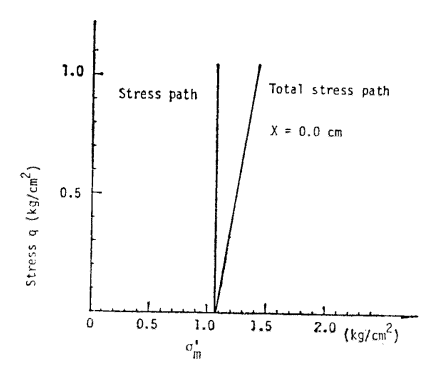

Fig. 12 Dynamic Stress Path (calculated Results)

tions in wave propagation. Fig. 8 and 9 correspond to Figs. 6 and 7 respectively. In each case, the stress-strain relation is bi-linear and the type of dissipation is hysteretic, but in Fig. 8, the viscoelastic effect of Voigt type turns up. That is to say, the strain has the delayed component. The new stress-strain relation can describe the behavior of cohesive soil in both loading part and unloading part. The stress-strain relation obtained in the stress wave propagation test. (Fig. 10) and the result obtained by Vey \& Strauss ${ }^{35}$ ) (Fig. 11) are similar to the calculated result. Fig. 12 shows the dynamical stress path obtained by calculation. The proposed stressstrain relation can describe the behavior of 
normally consolidated clay better than previously reported one. ${ }^{12)}$

\section{CONCLUSIONS}

The following main conclusions are obtained in the present research.

(1) From the Fourier transformation of the stress wave obtained in the wave propagation test, the cohesive soil seems to have a viscoelastic property at a low stress level.

(2) From a point of view of Green \& Naghdi, the theory of mixture of an elastic fluid and a viscoelastic-viscoplastic solid is proposed in order to explain the dynamic behavior of saturated cohesive soil. Moreover, by this theory, the physical meaning of Terzaghi's effective stress is rationally explained.

(3) The proposed constitutive theory can fairly express the test results obtained by the wave propagation test, especially, the stress-strain relation during the unloading.

The author wishes to thank Koichi Akai, Professor of Transportation Engng. of Kyoto University, for suggesting the present study and constructive criticism. The author is also indebted to Masayuki Hori, Research Associate of Transportation Engng. of Kyoto University for final support and helpful advices, and thanks to Kenji Kita, Graduate Student of Kyoto University for his support to numerical calculation.

\section{REFERENCES}

1) Biot, M. A.: Mechanics of Deformation and Acoustic Propagation in Porous Media, J. Appl. Phys., 33, 1962, pp. 1482-1498.

2) Ishihara, K.: Theory of Consolidation of a Porous Material with Heat Effect based on the Irreversible Thermodynamics, Trans. of JSCE, No. 113, Jan., 1965, pp. 28-42.

3) Truesdell, C.: Rational Thermodynamics, New York, McGraw-Hill, 1969.

4) Coleman, B. D. and W. Noll: Thermodynamics of Elastic Materials with Heat Conduction and Viscosity, Arch. Rational Mech. Anal., Vol. 13, 1963, pp. 167-178.

5) Green, A. E. and P. M. Naghdi: A Dynamical Theory of Interacting Continua, Int. J. Eng. Sci. Vol. 3, 1965, pp. 231-241.

6) Ingram J. D. and A. C. Eringen,: A Continuum Theory of Chemically Reacting Media-I, Int. J. Eng. Sci. Vol. 3, 1965, pp. 197-212.

7) Bowen, R. M.: Toward a Thermodynamics and Mechanics of Mixtures, Arch. Rational
Mech. Anal. Vol. 24, ; 1967, pp. 370-403.

8) Müller, I.: A Thermodynamic Theory of Mixtures of Fluids, Arch. Rational Mech. Anal. Vol. 28, 1968, pp. 1-39.

9) Ishihara, K.: Approximate Forms of Wave Equation for Water-Saturated Porous Materials and Related Dynamic Modulus, Soils and Foundations, Vol. 10, No. 4, 1970, pp. 10-38.

10) Akai, K. and M. Hori: Considerations of Wave Characteristics in Soil Assumed as a Viscoelastic Material, Proc. JSCE, No. 221, Jan., 1974, pp. 81-91.

11) Adachi, T.: Consideration of Constitutive Equation of Soil Material, Preprint, 27th Conf. JSCE, 1972, pp. 333-336. (in Japanese)

12) Akai, K. and F. Oka: Thermodynamic Theory of Inelastic Materials and its Application to Stress Wave Propagation in Cohesive Soil, Proc. JSCE, No. 253, Sept., 1976, pp. 109-122.

13) Kondner, R. L. and M. M. K. Ho: Viscoelastic Response of a Cohesive Soil in the Frequency Domain, Trans. Soc. of Rheology, Vol. 9, Part 2, 1965, pp. 329-342.

14) Nishigaki, $Y$. and T. Hirobe: Characteristics of Hysterisis of Osaka Diluvial Clays, Preprint, 8th Conf. JSME, 1973, pp. 359-362. (in Japanese)

15) Hatano, $T$. and $H$. Watanabe: Dynamic and Static Viscoelastic Constants and Poisson's Ratio of Clay, Sand and Crushed Stone, Proc. of JSCE, No. 164, April, 1969, pp. 33-49. (in Japanese)

16) Krizek, R. J. and A. G. Franklin: Energy Dissipation in a Soft Clay, Proc. Int. Symp. Wave Propagation and Dynamic Properties of Earth Materials, Univ. New Mexico, 1967, 1967, pp. 293-304.

17) Hara, A.: Dynamic Characteristics of Ground and its Application, Proc. Symp. 2nd Ground Motion, JSAE, 1973, pp. 3339. (in Japanese)

18) Parmelee, R. A., J. Penzien, C. F. Scheffey, H. B. Seed and G. R. Thiers: Seismic Effects on Structures Supported on Piles Extending through Deep Sensitive Clays, Report No. 64-2, Inst. of Eng. Res., Univ. of Calif., Barkeley, California, 1964.

19) Atkins, R. J. and R. E. Craine: Continuum Theory of Mixtures (Basic Theory and Historical Development), Q. J. Mech. Appl. Math., Vol. 29., 1976, pp. 209-244.

20) Truesdell, C. and R. Toupin: The Classical 
field Theories, in "Handbuch der Physik" (S. Flügge, ed) Vol. III/1, Springer-Verlag, Berlin, 1960.

21) Green, A. E. and P. M. Naghdi: Remarks on a paper by R. M. Bowen, Arch. Rational Mech. Anal. Vol. 27, 1967, pp. 175-180.

22) Green, A. E. and P. M. Naghdi: Entropy Inequalities for Mixtures, Quart. J. Mech. Appl., Vol. 24, 1971, pp. 473-485.

23) Green, A. E. and P. M. Naghdi: On Continuum Thermodynamics, Arch. Rational Mech. Anal., Vol. 48, 1972, pp. 352-378.

24) Akai, K. and T. Tamura: An Application of Nonlinear Stress-Strain relations to Multi-Dimensional Consolidation Problems, Disaster Privension Research Institute Annuals, No. 19. B-2, Kyoto Univ., 1976, pp. 15-29 (in Japanese)

25) Skempton, A. W.: Effective Stress in Soils, Concrete and Rocks, Pore Pressure and Suction in Soils, London Butterworths, 1961, pp. 4-16.

26) Lambe, T. W. and R. V. Whitman: Soil Mechanics, Jhon Wiely \& Sons, Inc., 1969, pp. 241-250.

27) Scott, R. F.: Principle of Soil Mechanics, Addison-Wiesly Pub. Company, Inc., 1963.

28) Mitchell, J. K.: Fundamental of Soil Behavior, John Wiely \& Sons, Inc., 1976, pp. 186-196.
29) Kenyon, D. E.: The Theory of an imcompressible Solid-Fluid Mixture, Arch. Rational Mech. Anal. Vol. 62. 1976, pp. 131-147.

30) Green, A. E. and T. R. Steel: Constitutive Equations for Interaction Continua, Int. J. Engng. Sci. Vol. 4, 1966, pp. 483-500.

31) Toki, K. \& T. Sato: One-Dimensional Accceleration Waves in a Mixture of 2 Phases, Theoretical and Applied Mechanics, Vol. 22, 1974, pp. 89-102.

32) Adachi, T. and Okano: A Constitutive Equations for Normally Consolidated Clay, Soils and Foundations, Vol. 14, No. 4, 1974, pp. 55-73.

33) Akai, K., M. Hori and T. Shimogami: Study on Stress Wave Propagation through Cohesive Soils by Means of Triaxial Shock Tube, Proc. JSCE, No. 228 August, 1974, pp. 99108.

34) Ishihara, K.: Fundamentals of Soil Dynamics, Kajima Pub., 1976, pp. 50-69. (in Japanese)

35) Vey, E. and L. V. Strauss: Stress-Strain Relationships in Clay due to Propagating Stress Waves, Proc. Int. Symp. on Wave Propagation and Dynamic Properties of Earth Materials, 1967, pp. 575-586.

(Received May 19, 1977) 